\title{
IDENTIFICACIÓN DE ACTIVIDADES ESTRATÉGICAS PARA LA GENERACIÓN DE EMPLEO EN COSTA RICA, UTILIZANDO LA MATRIZ INSUMO PRODUCTO
}

\author{
IDENTIFICATION OF STRATEGIC ACTIVITIES FOR THE GENERATION OF \\ EMPLOYMENT IN COSTA RICA, USING THE INPUT-OUTPUT MATRIX
}

\section{Greivin Hernández González ${ }^{1}$}

\begin{abstract}
Resumen
El objetivo del presente estudio es determinar las actividades económicas con mayor capacidad de generación de empleo en Costa Rica. La identificación de tales actividades se realiza estimando diferentes indicadores como generación de empleo total, indirecto, elasticidad empleo producto, entre otros. Esto permite a los hacedores de política pública contar con más herramientas para promover la actividad económica y el empleo. El estudio brinda pronósticos sobre el potencial de creación de empleo según actividad económica ante respuestas a aumentos en la demanda final. Adicionalmente, se compara la capacidad de generación de empleo de los sectores establecidos en regímenes especiales con los del régimen definitivo.
\end{abstract}

Palabras clave: elasticidad; zonas francas; régimen definitivo; encadenamientos; reactivación económica

\begin{abstract}
The objective of the study is to identify the economic activities with the greatest capacity to generate employment in Costa Rica. Such activities are identified by estimating different indicators such as generation of total and indirect employment and employment-output elasticity. This gives public
\end{abstract}

Doi: http://dx.doi.org/10.15359/eys.22-52.2

Fecha de recepción: 04-02-2017. Fechas de reenvíos: 13-02-2017, 06-03-2017, 12-06-2017, 16-07-2017. Aceptado el 22-07-2017. Publicado el 26-07-2017.

${ }^{1}$ Economista. Académico de la Escuela de Economía de la Universidad Nacional (UNA), Costa Rica. Investigador del Observatorio de la Coyuntura Económica y Social de la Escuela de Economía de la Universidad Nacional (UNA), Costa Rica. El autor agradece los valiosos comentarios de Andrea Zúñiga Rojas al borrador de esta investigación. Correo electrónico: greivin.hernandez.gonzalez@una.cr

Greivin Hernández González

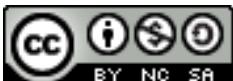

Revista Economía y Sociedad by Universidad Nacional is licensed under a CreativeCommons Reconocimiento-NoComercial- 
policy makers more tools to promote economic activity and employment. The study provides forecasts on the potential creation of employment by economic activity, in response to increases in final demand. In addition, the capacity to generate employment of sectors established in special regimes is compared with those of the definitive regime.

Keywords: elasticity; free zones; definitive regime; linkages; economic reactivation

\section{Introducción}

A partir de la Gran Recesión causada por la crisis financiera internacional de 2008, Costa Rica ha enfrentado un serio problema de desempleo. Según las cifras de la Encuesta Continua de Empleo (Instituto Nacional de Estadística y Censos (INEC), 2016), a partir del tercer trimestre de 2010 y hasta el mismo momento de 2016 , la tasa de desempleo abierta ha sido en promedio de $9,7 \%$, alcanzando niveles de hasta el 10,9\%, y sin caer más allá de 8,3\%.

Dicho nivel de desempleo se considera anormalmente alto, por cuanto según la Encuesta de Hogares de Propósitos Múltiples el desempleo promedió el 5,5\% de la población económicamente activa entre 2005 y 2008 (en 2009 saltó a 7,8\%\%) (INEC, 2009). Aún más, si se analizan otros indicadores del mercado de trabajo, es evidente que la economía está siendo absolutamente incapaz de crear los empleos suficientes para absorber a la población que busca laborar, y esto está afectando las condiciones de trabajo de algunos sectores de la población ocupada.

Muestra de ello es que, si se toma en cuenta a la población disponible desalentada ${ }^{2}$ o a la disponible con limitaciones ${ }^{3}$, sería posible estimar tasas de desempleo ampliadas que indican que este flagelo podría rozar el 20\% (Morales, Hernández, Salazar, \& Zárate, 2017), dado que existen muchas personas en edad de trabajar y con capacidad de buscar empleo activamente, que están fuera de la fuerza laboral. Dichas personas podrían sumarse a la fuerza laboral en cualquier momento, con lo que presionarían al alza la tasa de desempleo.

La tendencia en materia de empleo en los últimos años es hacia una fuerte reducción de tasa de ocupación, dado que muchas personas se salen del mercado de trabajo desanimadas por la falta de oportunidades para laborar, combinado con un estancamiento y, en ocasiones, reducción del número de ocupados. A partir del tercer trimestre de 2015, los cambios en la tasa de desempleo

\footnotetext{
2 Personas con edad para trabajar e interés de hacerlo pero que no buscan empleo por diferentes circunstancias como falta de dinero, agotamiento de la búsqueda, discriminación de etnia, sexo, edad, discapacidad, etc.

${ }^{3}$ Personas con edad para trabajar y con interés de hacerlo que no buscan trabajo activamente puesto que poseen algún tipo de limitación como enfermedad, accidente, educación, obligaciones personales, familiares, etc. 26
}

Greivin Hernández González

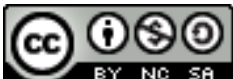

Revista Economía y Sociedad by Universidad Nacional is licensed under a CreativeCommons Reconocimiento-NoComercial- 
abierta se pueden clasificar como "reducciones no dinámicas", situación que ocurre cuando la disminución en la tasa mencionada se explica por una caída en la fuerza laboral más que por un aumento en el número de ocupados.

La situación apuntada se replica en el caso de los datos de subempleo y empleo informal, en los que las tasas son altas y tienden a mejorar no por el surgimiento de mayores oportunidades de empleo, sino porque se registra una caída en la oferta de trabajo. Las causas de tal situación son coyunturales, como la citada crisis, pero las hay también estructurales, y tienen que ver con deficiencias tanto de la oferta como de la demanda de trabajo. Estudios señalan aspectos de la demanda como el bajo crecimiento económico; la carencia de encadenamientos productivos de los sectores más dinámicos de la economía; pérdida de competitividad de sectores empleadores importantes (como el agrícola y algunas actividades de servicios); la fuerte reducción en el empleo público; así como elementos de la oferta tales como baja escolaridad, insuficiente productividad laboral; y otros como el cambio tecnológico y el desalineamiento entre la oferta y la demanda de trabajo (Hernández y Villalobos, 2016). En este último punto, en numerosas ocasiones el sector privado ha manifestado la necesidad de contar con miles de profesionales en áreas en las que carecen de personal suficiente (REPRETEL, 2016).

Por lo anterior, el presente estudio considera oportuno investigar sobre posibles medidas de política que pudiesen fomentar la creación de empleos. Bajo esta perspectiva, es posible identificar dos grandes áreas de trabajo, la primera relacionada con las políticas de reactivación económica, y la segunda centrada en las políticas de empleo, sean estas pasivas o activas. Las pasivas incluyen medidas de atención paliativa a la población desempleada; las activas están constituidas por: a) programas de búsqueda de empleo, b) capacitación y c) financiamiento a la creación de empleos, sean estos en el sector público o privado.

Esta investigación se enmarca dentro de la primera área de política pública, que tiene que ver con la preocupación de relacionar el crecimiento económico con la generación de empleos. En particular, se busca identificar aquellas actividades productivas estratégicas en materia de empleo, tanto por su capacidad de generar oportunidades de trabajo directo como indirecto. Además, se realiza un análisis diferenciado para las actividades radicadas en regímenes especiales (zonas francas), dado que la fuente de los datos permite hacer esta identificación.

El documento continúa en la sección dos, en la que se aborda la metodología utilizada y las fuentes de información; en la sección tres se presentan los resultados obtenidos y se elabora una serie de indicadores de creación de empleos a partir de cambios en la demanda final según actividad económica; finalmente, en la sección cuatro se realiza una discusión de los resultados. 


\section{Metodología}

La presente investigación tiene un enfoque cuantitativo, dado que se fundamenta en la utilización de bases de datos a partir de las cuales se crearán indicadores numéricos que buscan establecer categorías definidas de sectores estratégicos para la generación de empleos. Dentro de este enfoque, se plantea una investigación de alcance correlacional, por cuanto se explicará y cuantificará la relación entre variables, además de que se ofrecerán predicciones del comportamiento de las mismas.

La principal fuente de información es la matriz insumo producto (MIP) elaborada por el Banco Central de Costa Rica (BCCR) para el año 2012 (BCCR, 2016), la cual es la más reciente hasta ahora publicada por dicha entidad, y la más detallada, pues cuenta con una desagregación de 128 actividades económicas ${ }^{4}$ (la anterior contaba con 77 actividades).

Una matriz insumo producto constituye una representación numérica de la economía en un momento dado, por consiguiente, se considera un modelo de equilibrio general estático. Su objetivo fundamental es representar la interdependencia de las industrias, de manera que las entradas de unas (insumos) son las salidas de otras (productos). Cada columna representa el valor monetario de los insumos por actividad económica, mientras que las filas determinan el valor de la producción.

El modelo de la matriz insumo producto fue formalizado por el economista de origen alemán Wassily Leontief (1906-1999), quien en 1973 recibió el Premio Nobel de Economía a propósito de la misma. Leontief, a su vez, se basó en los aportes de Francois Quesnay, León Walras y Calos Marx para la elaboración inicial de la MIP.

Adicionalmente, la MIP 2012 elaborada por el Banco Central de Costa Rica presenta una identificación parcial de actividades en regímenes "especial" o "definitivo". No es total por cuanto existen 20 actividades para las que no se determina su régimen y que en conjunto suman el $12 \%$ de la producción de la economía y el 7\% del empleo directo.

Para la estimación de sectores estratégicos en la generación de empleo, se considera estimar la generación de empleo total; es decir, la sumatoria del empleo directo y el indirecto. El dato de empleo directo se obtiene del vector de empleo de la MIP 2012; sin embargo, el cálculo de empleo indirecto requiere de un tratamiento de la misma mediante técnicas de álgebra matricial.

\footnotetext{
${ }^{4}$ Si bien la codificación de la MIP parece indicar que existen 136 actividades, la agrupación de algunas de ellas hace que en la práctica sea el número indicado. 28

Greivin Hernández González

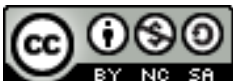

Revista Economía y Sociedad by Universidad Nacional is licensed under a CreativeCommons Reconocimiento-NoComercial- 
Siguiendo la metodología utilizada por Sánchez (2014), el primer paso consiste en estimar la matriz de coeficientes técnicos, esto es, la proporción de requerimientos que representan todas las actividades dentro de una industria determinada, o lo que es lo mismo:

$A=\left(\left(\begin{array}{ccc}\frac{a_{11}}{x_{1}} & \cdots & \frac{a_{1 n}}{x_{n}} \\ \vdots & \ddots & \vdots \\ \frac{a_{n 1}}{x_{1}} & \cdots & \frac{a_{n n}}{x_{n}}\end{array}\right)\right)$

Donde:

A =matriz de coeficientes técnicos

$a_{i j}=$ ventas intermedias del sector $\mathrm{i}$ al sector $\mathrm{j}$

$x_{j}=$ producción del sector $\mathrm{j}$

Posteriormente, se calcula la matriz de Leontief, que muestra los coeficientes técnicos de requerimientos directos e indirectos de los " $\mathrm{j}$ " sectores sobre los "i" sectores. La misma tiene la siguiente forma:

$M L=(I-A)^{-1}$

Donde:

$\mathrm{ML}=$ matriz de requerimientos técnicos de Leontief

I = matriz identidad

Seguidamente, se obtiene el vector de requerimientos de empleo directo, dividiendo el vector de empleo entre la producción de cada sector, de la siguiente manera:

$L=\left(\frac{l_{1}}{x_{1}}, \ldots, \frac{l_{j}}{x_{j}}\right)$

Donde:

$\mathrm{L}=$ vector de requerimientos de empleo directo

$l_{j}=$ personal ocupado por el sector $\mathrm{j}$

$x_{j}=$ producción del sector $\mathrm{j}$

Luego, se procede a obtener la matriz de requerimientos directos e indirectos de empleo. Para ello, es preciso diagonalizar el vector "L" y multiplicarlo por (2), o lo que es lo mismo:

$H=L d * M L$ 
Donde:

$\mathrm{H}=$ matriz de requerimientos directos e indirectos de empleo

$\mathrm{Ld}=$ matriz con la diagonalización del vector $\mathrm{L}$

Finalmente, dado que las entradas de la matriz $\mathrm{H}$ representan los coeficientes de requerimientos directos e indirectos de empleo, se suman los coeficientes por columnas para obtener el requerimiento de empleo total para cada sector. Al multiplicar cada coeficiente por el vector de producción, es posible obtener el empleo total de cada sector " $\mathrm{j}$ ". El empleo indirecto es la diferencia entre el empleo total estimado y el vector de empleo directo.

Por otra parte, el cálculo de la elasticidad empleo producto tiene la siguiente ecuación:

$E_{j}^{e}=\left[\sum_{i=1}^{n}\left(\frac{l_{i}}{x_{i}}\right) b_{i j}\right] \frac{f_{i}}{L}$

Donde:

$E_{j}^{e}=$ vector de elasticidades empleo producto de los $\mathrm{j}$ sectores

$l_{i}=$ empleo directo del sector $\mathrm{i}$

$X_{i}=$ producción del sector $\mathrm{i}$

$b_{i j}=$ coeficiente ij-ésimo de la matriz $\mathrm{H}$ calculada en (4)

$f_{i}=$ demanda final del sector $\mathrm{i}$

$\mathrm{L}=$ empleo total de la economía

\section{Resultados}

Los cálculos realizados según la metodología descrita en la sección dos señalan que las cuatro actividades que más generan empleo son: comercio; construcción de edificios; actividades de servicio de comida y bebidas; y enseñanza (ver Tabla 1 en Anexo), quienes conjuntamente explican el $29 \%$ del empleo total de la economía. El cálculo toma en cuenta tanto el empleo directo como el indirecto, ver Figura $\underline{1}$. 


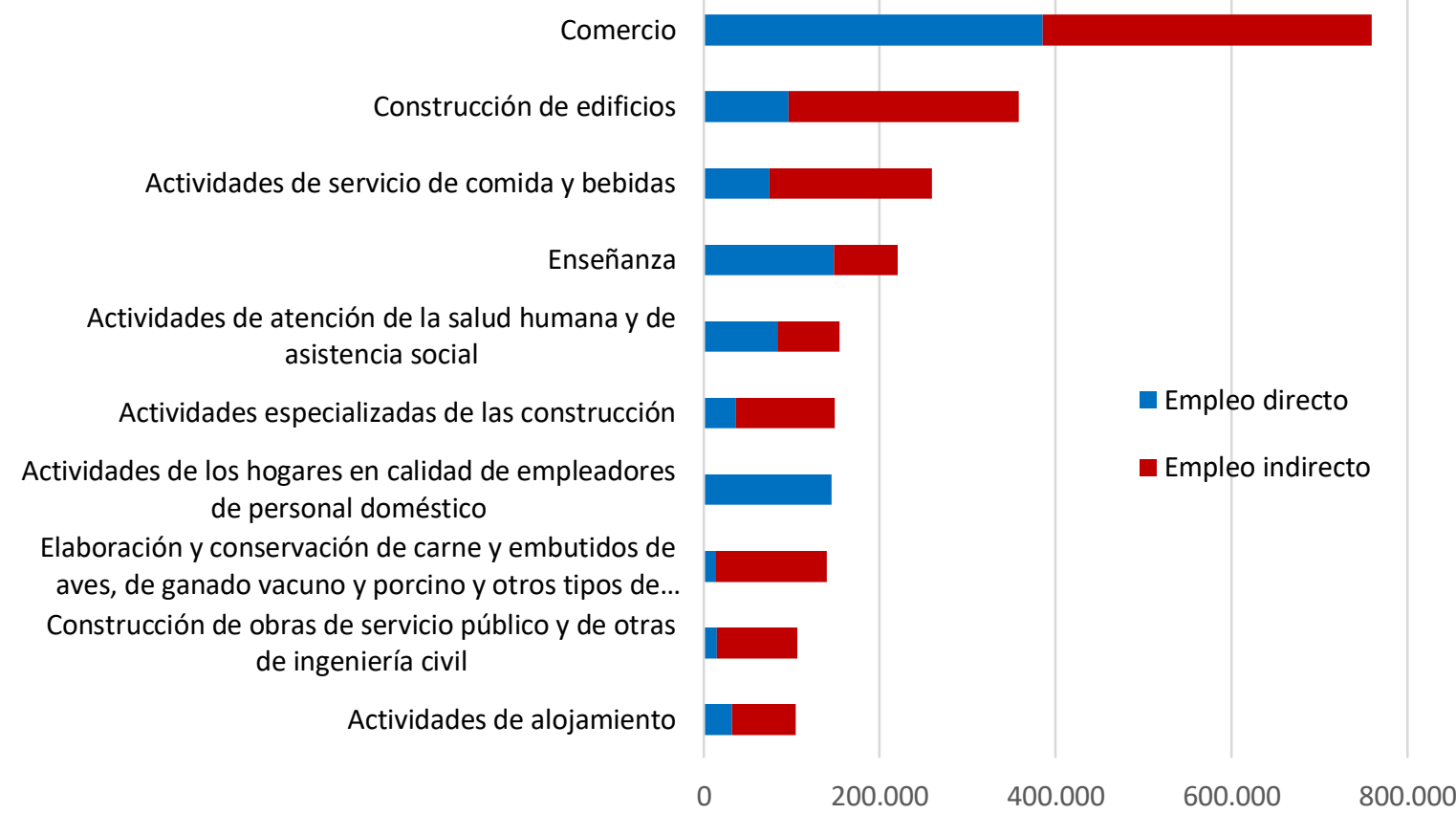

Figura 1. Costa Rica: Principales actividades económicas generadoras de empleo directo e indirecto, 2012 (\# de ocupados). Fuente: elaboración propia.

Por su parte, las actividades que generan más empleo indirecto en términos de volumen son: comercio (11\%); construcción de edificios (8\%); actividades de servicio de comida y bebidas (6\%); y elaboración y conservación de carne y embutidos de aves, de ganado vacuno y porcino y otros tipos de carne (4\%).

En términos de la generación de empleo indirecto, también conviene conocer la relación empleodirecto/empleo-indirecto para determinar las actividades que más empleo indirecto generan en términos comparables, eliminando la distorsión que conlleva la escala de producción. En este sentido, las tres actividades económicas que generan más empleos indirectos en relación con los empleos directos son: elaboración de café oro; elaboración de aceites y grasas de origen vegetal y animal; y transporte por ferrocarril. Como muestra la Figura 2 , en el caso de la elaboración de café oro, el $98 \%$ del empleo total generado es indirecto, por lo que se puede decir que por cada empleo generado al interior del sector, se generan 42 empleos en otras actividades. Un análisis detallado del empleo indirecto generado para todas las actividades económicas muestra que son las actividades de los sectores agrícolas, agroindustrial y alimentario las que más empleos indirectos generan, ver Tabla $\underline{2}$ del Anexo. 


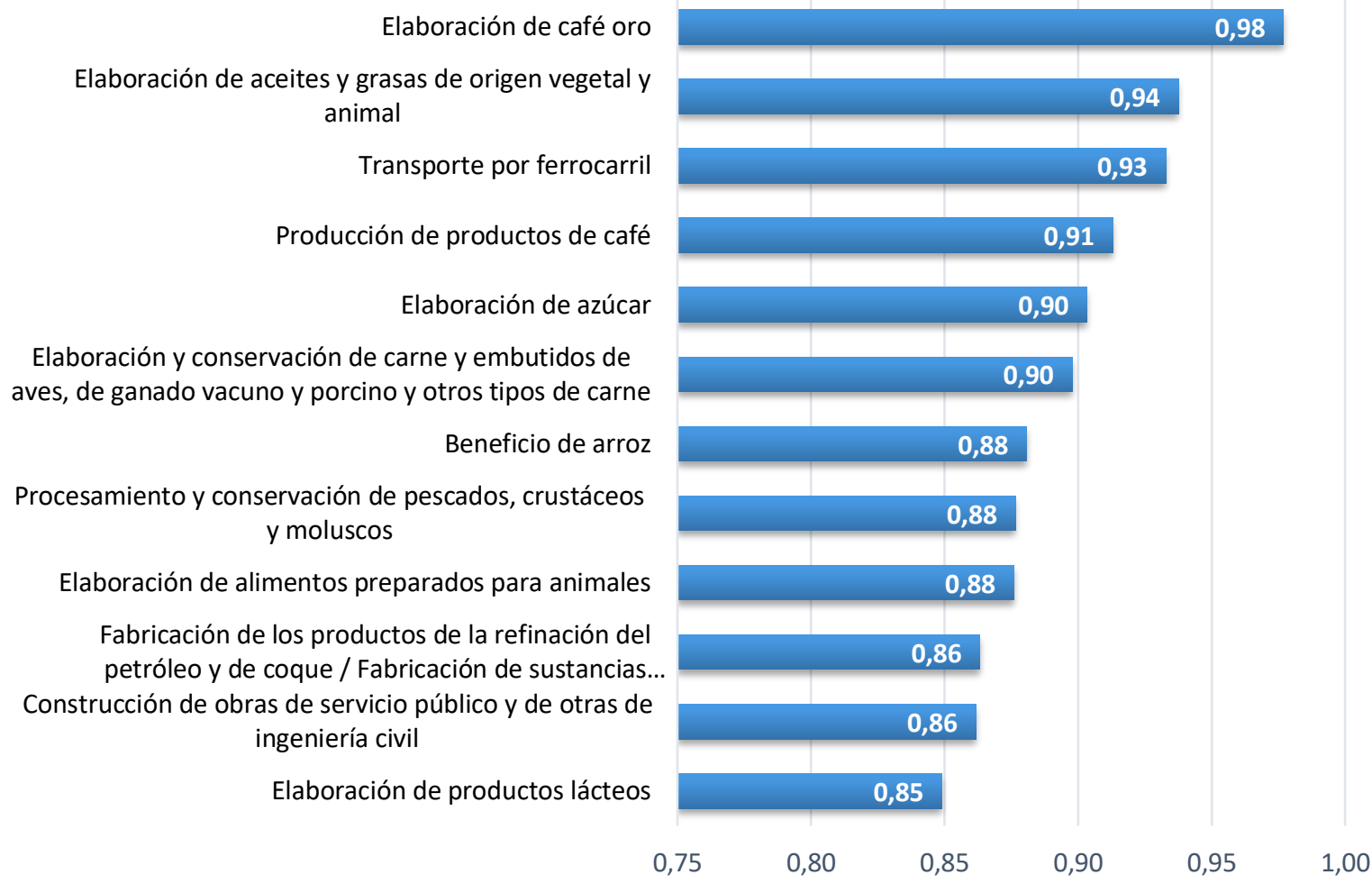

Figura 2. Costa Rica: actividades con mayor generación de empleo indirecto relativo, 2012 (proporción de empleo indirecto dentro del empleo total). Fuente: elaboración propia.

En torno a lo anterior, cabe indicar que, si bien es cierto las actividades económicas que más empleo indirecto relativo generan pueden no ser muy representativas en términos de la generación de empleo total, se consideran estratégicas por cuanto crean una gran cantidad de empleos indirectos. La razón de este comportamiento es su alto nivel de encadenamientos con otras actividades al interior de la economía.

El siguiente indicador que conviene comentar es la elasticidad empleo-producto. Este cálculo permite determinar cuánto varía el empleo en el sector "j" ante cambios exógenos en la demanda final de dicho sector "j". Las actividades económicas con coeficientes de elasticidad más altos son: comercio $(0,37)$, construcción de edificios $(0,17)$, actividades de servicio de comida y bebidas $(0,12)$, y enseñanza $(0,11)$ (ver 1 en Anexo). Dicho resultado es coincidente con el cálculo del empleo total.

El resultado del coeficiente de elasticidad permite pronosticar cambios en el empleo ante cambios en la demanda final de producto, ceteris paribus. Por ejemplo, la Figura $\underline{3}$ muestra el cambio en el número de ocupados por sector ante un aumento de $1 \%$ en la demanda final para las 10 actividades económicas con los coeficientes más altos. De esta manera, es posible afirmar 32

Greivin Hernández González

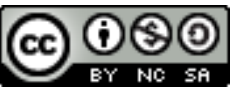

Revista Economía y Sociedad by Universidad Nacional is licensed under a CreativeCommons Reconocimiento-NoComercial- 
que, según la estructura económica costarricense de 2012, como resultado de un aumento de $1 \%$ en la demanda final del sector comercio, la economía es capaz de crear 7595 empleos en dicho sector.

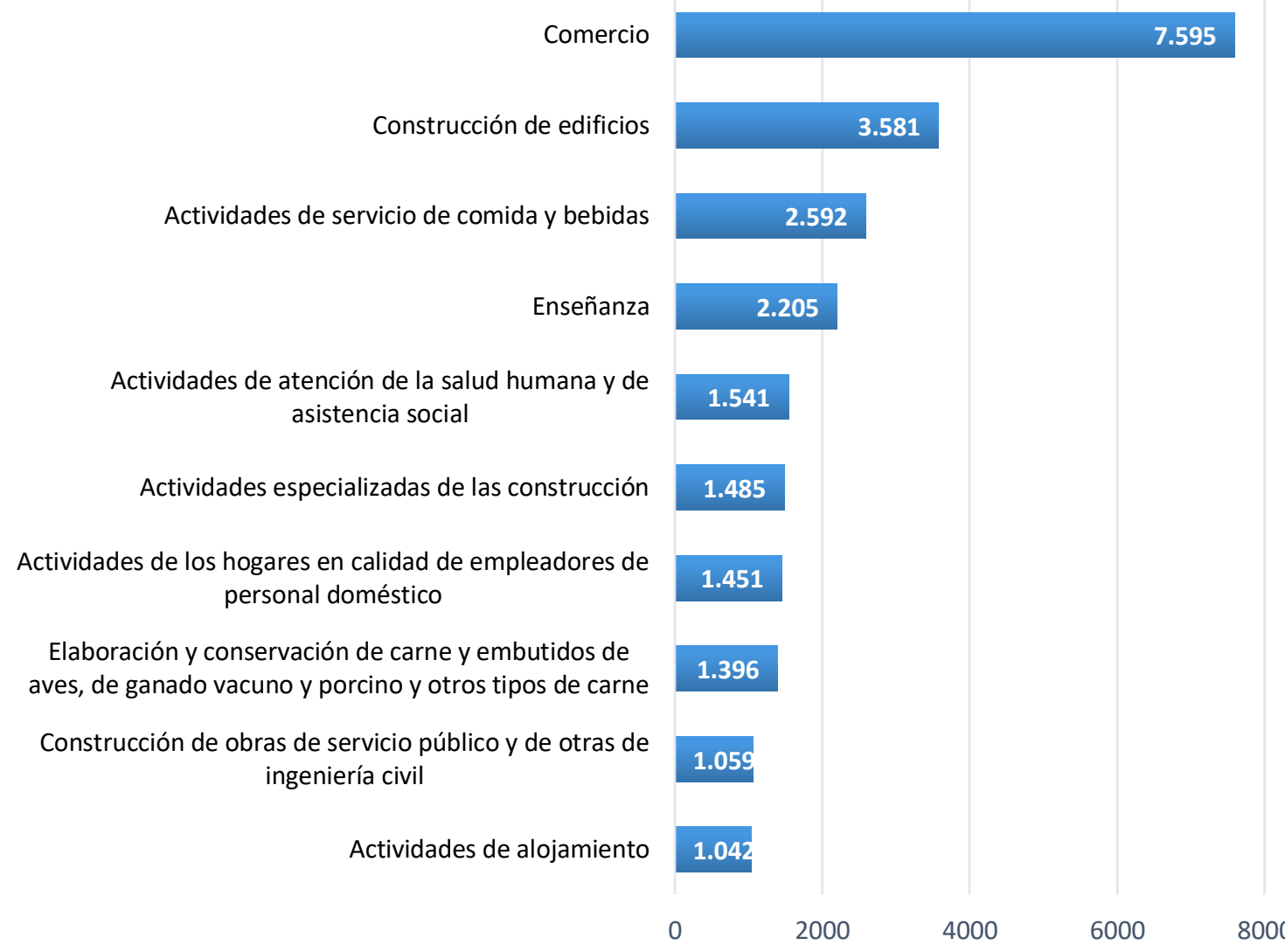

Figura 3. Costa Rica: empleos generados por un aumento de $1 \%$ en la demanda final según sector, 2012 (Número de ocupados). Fuente: elaboración propia.

Los cálculos realizados también permiten determinar cuáles son los empleos más "asequibles" para el Estado en términos de política pública; es decir, los empleos que es posible generar por la vía de un aumento en la demanda final de alguna actividad en particular. De esta manera, se desprende que las cinco actividades que más empleo generan con un menor costo en términos de estímulo de la demanda son: cultivo de frijol, cultivo de maíz, actividades de los hogares en calidad de empleadores de personal doméstico, extracción de sal y cultivo de otras hortalizas, raíces o tubérculos (ver Tabla $\underline{3}$ en Anexo).

Como muestra la Figura 4 , el gasto público para generar un empleo por la vía del estímulo a la producción en la actividad de cultivo de frijol es de 1,2 millones de colones, mientras que para el caso de la fabricación de cuero y productos conexos excepto calzado es de 3,3 millones de colones. 


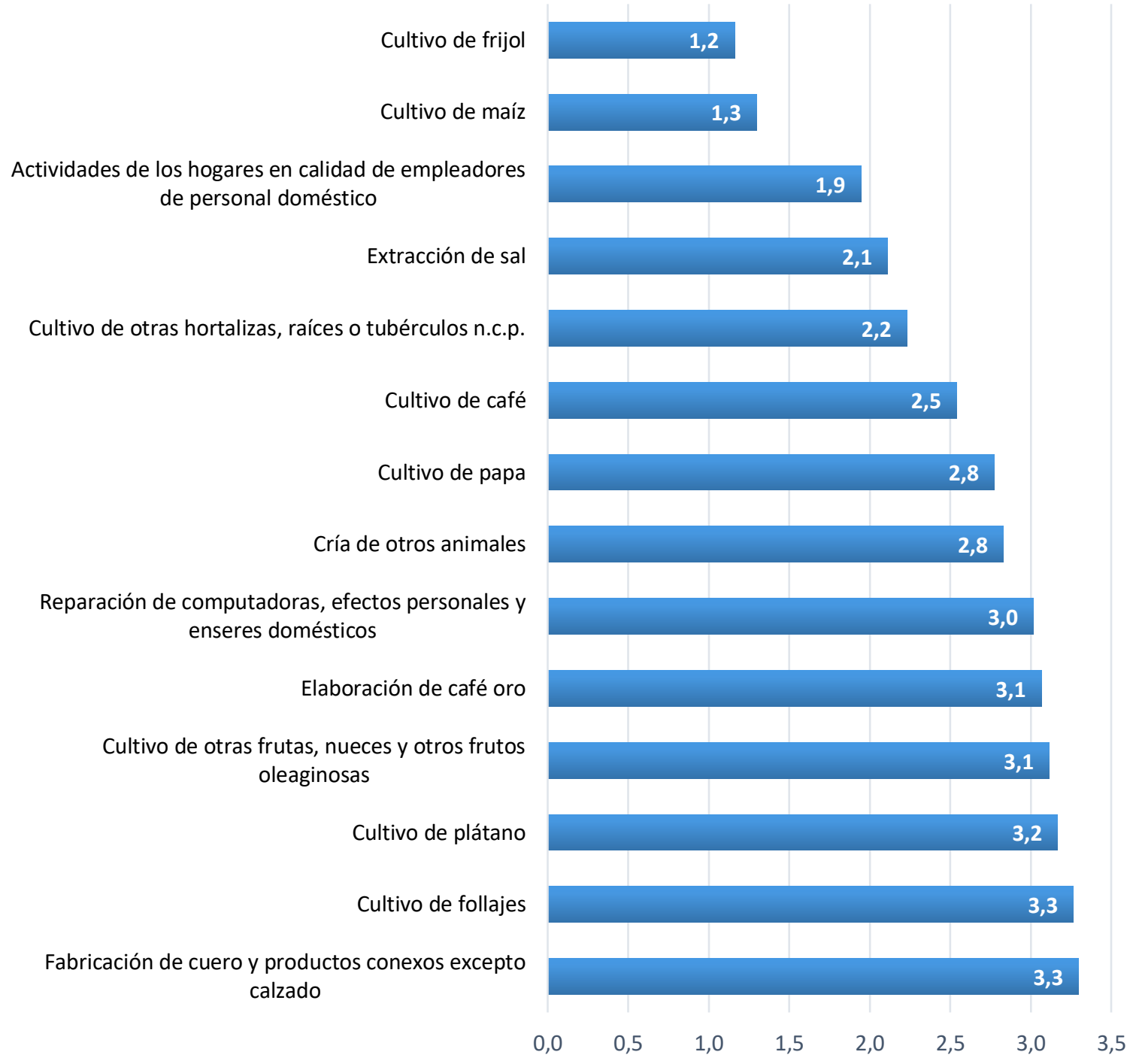

Figura 4. Costa Rica: gasto por empleo generado según actividad, 2012 (millones de colones). Fuente: elaboración propia.

Como novedad, la MIP 2012 permite empezar a analizar algunas características del empleo según sea su régimen de producción, especial o definitivo. Cabe insistir en que el análisis es parcial, por cuanto para 20 actividades no existe tal desagregación.

En torno a las 32 actividades localizadas en regímenes especiales, estas generaron 72119 empleos directos en 2012 (3\% del empleo total), siendo las más grandes generadoras de empleo directo: actividades administrativas y de apoyo de oficina y otras actividades de apoyo a las empresas; fabricación de instrumentos y suministros médicos y dentales; y actividades de consultoría en gestión financiera, recursos humanos, mercadeo, oficinas principales y afines. 34 
Por su parte, las más importantes en términos de empleo total son: fabricación de instrumentos y suministros médicos y dentales; actividades administrativas y de apoyo de oficina y otras actividades de apoyo a las empresas, y procesamiento y conservación de frutas y vegetales. El surgimiento de las dos últimas se debe a que están más encadenadas con el resto de la economía, por lo que son capaces de generar más empleos indirectos.

En cuanto a la generación de empleo indirecto en términos comparativos (como proporción del empleo total), las actividades en regímenes especiales que más encadenadas están a la economía son: elaboración de comidas, platos preparados y otros productos alimenticios $(0,92)$; procesamiento y conservación de pescados, crustáceos y moluscos $(0,87)$; y actividades inmobiliarias $(0,87)$ (ver Figura $\underline{5}$ ).

El resultado de los coeficientes de elasticidad para las actividades en regímenes especiales es coincidente con la generación de empleo total, con lo que las actividades que más generan empleo directo e indirecto de manera conjunta son las que mayor elasticidad empleo-producto presentan. Cabe agregar, que carece de sentido realizar el ejercicio del costo por empleo generado desde el punto de vista de estímulo a la demanda final por parte del gobierno costarricense, dado que las actividades económicas establecidas en regímenes especiales producen para el mercado externo.

La siguiente comparación que conviene realizar es la capacidad de generación de empleo de las actividades entre regímenes. Para ello, se utiliza la media aritmética de las 32 actividades en regímenes especiales vis $a$ vis las 108 que reportan empleo en el régimen definitivo ${ }^{5}$. De esta manera, se tiene que, en términos generales, las actividades en régimen definitivo tienen una elasticidad empleo más alta que las de regímenes especiales (0,0210 vs 0,0038 como promedio simple). Sin embargo, los sectores en regímenes especiales presentan un $71 \%$ de empleo indirecto como porcentaje del empleo total generado, mientras que las actividades económicas en el régimen definitivo generan un $60 \%$ de empleo indirecto como porcentaje del total. Esto muestra que existe un número importante de actividades bajo el régimen definitivo que están poco encadenadas al resto de la economía 6 .

\footnotetext{
${ }^{5}$ Existen 68 actividades que solo registran empleo en el régimen definitivo, seis actividades que solo lo tienen en regímenes especiales, 32 actividades que registran empleo en ambos regímenes y 28 para las que no se reporta empleo bajo ningún régimen.

${ }^{6}$ En total 41 actividades económicas generan menos empleos indirectos que el promedio de la economía, lo cual implica que realizan pocas compras intermedias locales para producir sus bienes y servicios finales.
} 


\author{
Elaboración de comidas, platos preparados y otros \\ productos alimenticios \\ Procesamiento y conservación de pescados, crustáceos y \\ moluscos \\ Actividades inmobiliarias \\ Fabricación de metales comunes \\ Procesamiento y conservación de frutas y vegetales \\ Destilación, rectificación, mezcla de bebidas alcohólicas y \\ vinos / Elaboración de bebidas malteadas, de malta, \\ bebidas no alcohólicas, aguas minerales, y otras aguas \\ embotelladas / Elaboración de productos de tabaco \\ Manipulación de carga y otras actividades de apoyo al \\ transporte
}

Fabricación de productos de caucho

Fabricación de productos textiles

Fabricación de plásticos y de caucho sintético en formas primarias / Fabricación de productos de plástico

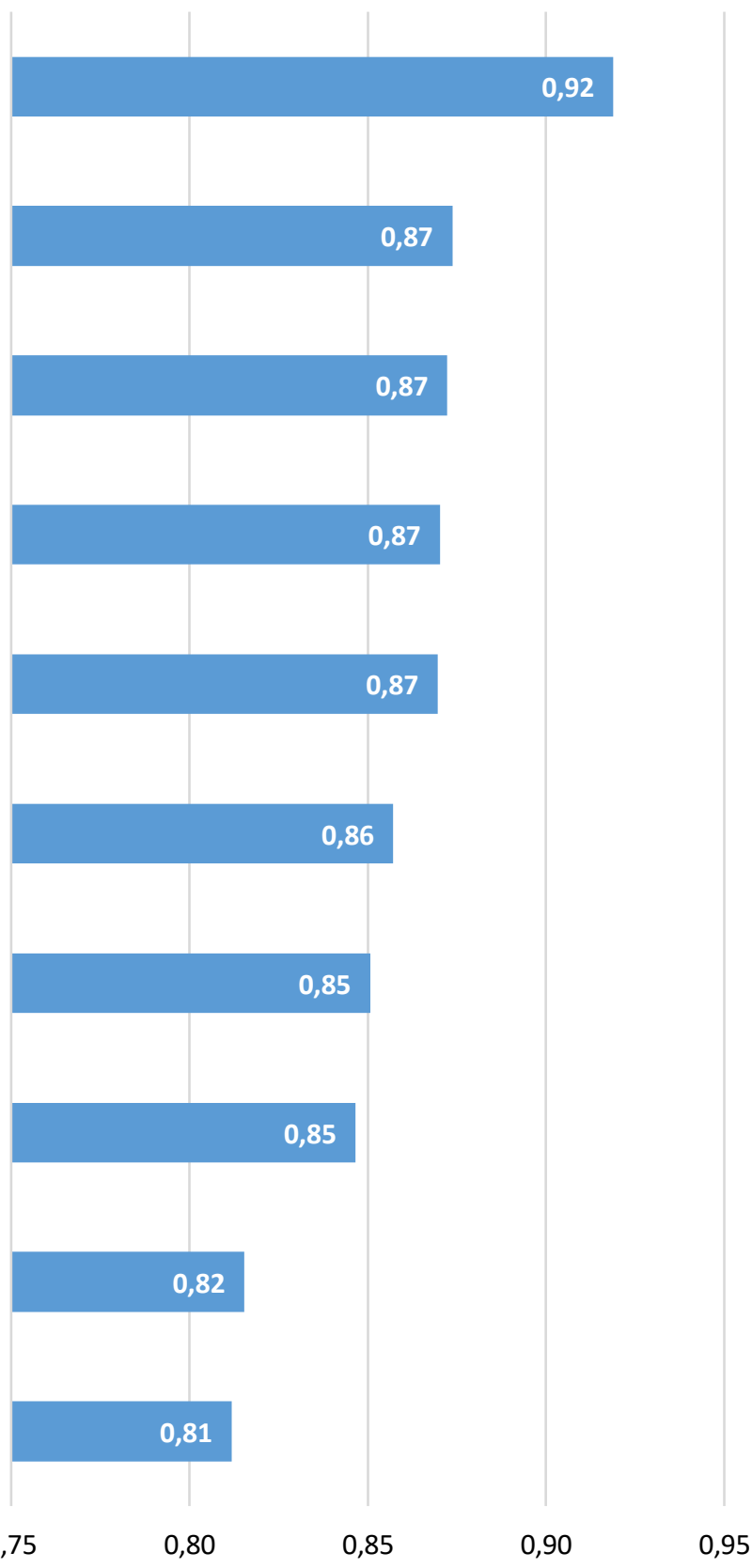

Figura 5. Costa Rica: Actividades bajo regímenes especiales con mayor generación de empleo indirecto, 2012 (proporción de empleo indirecto dentro del empleo total). Fuente: elaboración propia.

Por otra parte, si se compara el empleo directo por unidad de producto, resulta que las actividades de regímenes especiales generan 0,03 empleos por cada millón de colones producido, 36

Greivin Hernández González

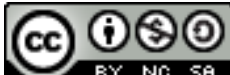

Revista Economía y Sociedad by Universidad Nacional is licensed under a CreativeCommons Reconocimiento-NoComercial- 
mientras que las del régimen definitivo, 0,06. El resultado quiere decir que, en promedio, para generar un empleo en los sectores bajo regímenes especiales es preciso una demanda final de 38 millones de colones, mientras que para las actividades en régimen definitivo, 17 millones de colones. Este resultado probablemente esté asociado a la mayor calificación y remuneración de los empleos establecidos en los regímenes especiales.

Cuando se comparan únicamente las 32 actividades que producen en ambos regímenes, los resultados son similares a los del análisis global. En primer lugar, la capacidad de generación de empleos indirectos sigue siendo más alta en las actividades en regímenes especiales; sin embargo, cabe anotar que la diferencia se acorta, dado que el empleo indirecto de las actividades en régimen definitivo subió a 0,65.

En segundo lugar, la elasticidad empleo-producto de los sectores en regímenes especiales es menor a la de los que se acogen al régimen definitivo, 0,0038 y 0,0086, respectivamente, ver Figura $\underline{6}$. Finalmente, el empleo generado por unidad de producto es levemente superior cuando la actividad se establece bajo el régimen definitivo: alcanza un valor de 0,027 para las actividades en regímenes especiales y de 0,032 para las del régimen definitivo. 
Fabricación de instrumentos y suministros médicos y dentales Actividades administrativas y de apoyo de oficina y otras...

Procesamiento y conservación de frutas y vegetales Actividades de consultoría en gestión financiera, recursos... Procesamiento y conservación de pescados, crustáceos y... Elaboración de comidas, platos preparados y otros...

Fabricación de prendas de vestir Fabricación de equipo eléctrico y de maquinaria n.c.p. Fabricación de productos de caucho Servicios de información, programación y consultoría... Fabricación de metales comunes Otras industrias manufactureras Actividades inmobiliarias Fabricación de productos textiles Fabricación de plásticos y de caucho sintético en formas... Fabricación de productos elaborados de metal, excepto...

Fabricación de vehículos automotores, remolques y... Actividades de investigación científica y desarrollo Manipulación de carga y otras actividades de apoyo al... Fabricación de muebles Cultivo de otros cereales, legumbres y semillas oleaginosas... Reparación e instalación de maquinaria y equipo Otras actividades profesionales, científicas y técnicas Reparación de computadoras, efectos personales y enseres... Pesca marítima y de agua dulce Destilación, rectificación, mezcla de bebidas alcohólicas y... Cultivo de otras hortalizas, raíces o tubérculos n.c.p. Cría de otros animales Almacenamiento y depósito Fabricación de vidrio y de productos de vidrio Propagación de plantas Cultivo de otras plantas no perennes y perennes

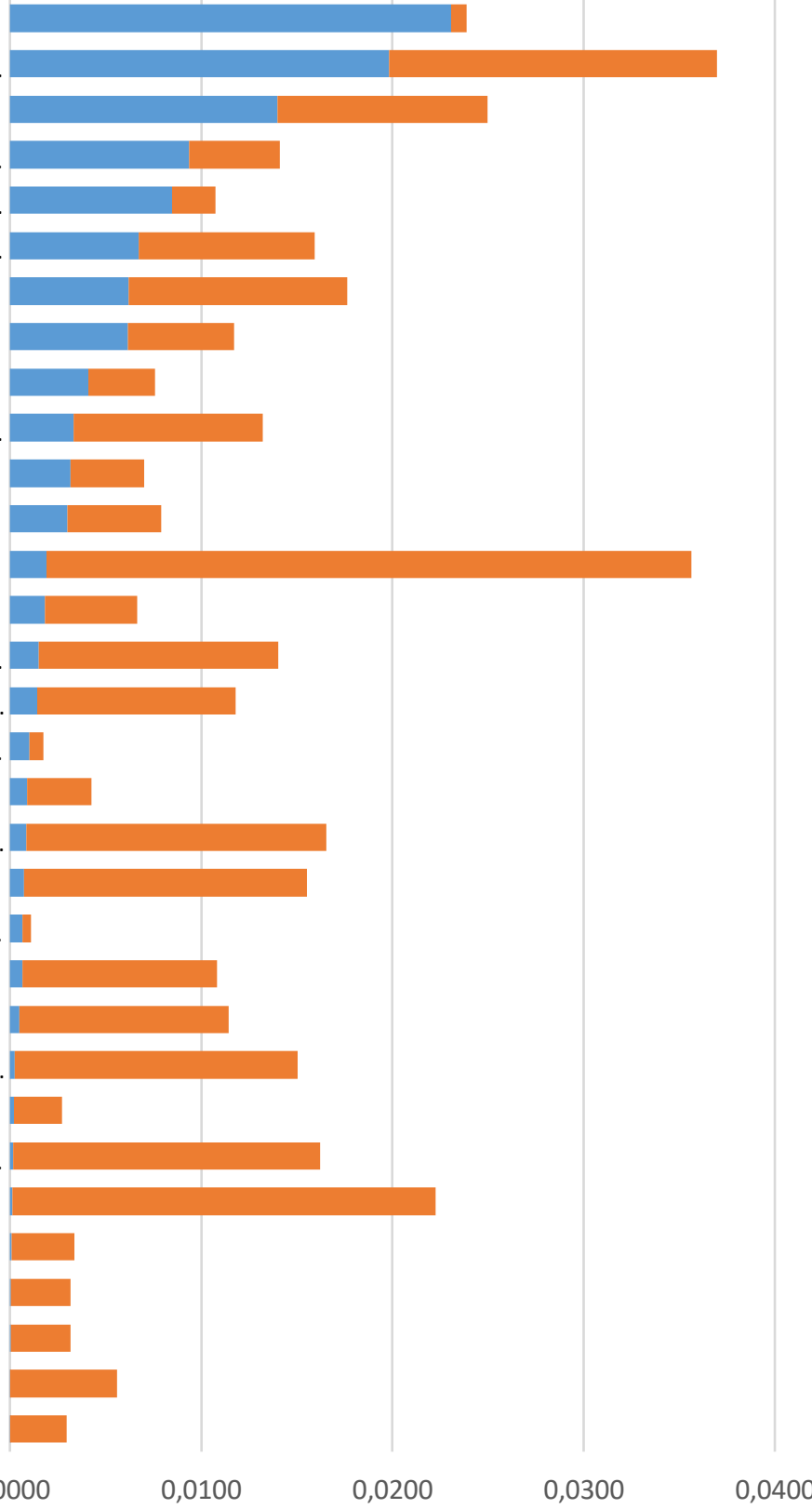

- Regímenes Especiales

Régimen Definitivo

Figura 6. Costa Rica: Elasticidad empleo-producto de las 32 actividades económicas con actividad identificada en regímenes especiales, según régimen, 2012. Fuente: elaboración propia. 


\section{Discusión}

El estudio realizado permitió identificar y jerarquizar sectores estratégicos de la economía costarricense para la generación de empleo según diferentes criterios. El ámbito estratégico de cada actividad económica se determinó considerando su volumen, su grado de generación de empleos indirectos (encadenamientos) o su costo relativo en términos de respuesta a cambios en la demanda final de producto.

Esto puede tener relevancia desde el punto de vista de la política económica, dado que permite a los hacedores de política pública identificar los sectores que más inciden en las cifras globales de empleo, facilita la focalización de políticas de promoción de empleo y la priorización del gasto público orientado a la generación de puestos de trabajo. Adicionalmente, se estimaron coeficientes que permiten pronosticar cambios en el número de empleados por actividad económica, según el grado de estímulo a la demanda final.

Como novedad de los estudios de empleo que hacen uso de la MIP, se realizó una comparación entre los regímenes de producción por actividad económica, el cual permitió determinar con precisión que las actividades en regímenes especiales tienen menor elasticidad empleo-producto y generan menos empleos por cada colón producido. A pesar de ello, el porcentaje de empleo indirecto respecto al empleo total generado por las actividades en regímenes especiales es en promedio mayor que el de todas las actividades establecidas en régimen definitivo.

Los hallazgos comentados implican que existe un amplio margen para generar empleo indirecto por parte de los sectores en regímenes especiales, así como falta de encadenamientos de numerosas actividades en régimen definitivo. Existe literatura reciente con una buena discusión sobre políticas de encadenamientos productivos y laborales; el diagnóstico para Costa Rica es que sus esfuerzos han sido débiles en comparación con los países más exitosos (Crespi, FernándezArias y Stein, 2014 y Salazar-Xirinachs, Nubler y Kozul-Wrigth, 2014).

Cabe realizar dos aclaraciones respecto a los resultados anteriormente apuntados. Primero, que las actividades han sido jerarquizadas como estratégicas tomando en cuenta únicamente la cantidad de empleos generados; no se utilizan otros criterios como el valor agregado aportado a la economía, el ingreso medio o la calidad del empleo. Segundo, se debe llamar la atención acerca de los supuestos de los modelos de insumo producto que pueden generar diferencias entre los pronósticos y los resultados de una política. Por ejemplo, los modelos asumen que las relaciones técnicas entre insumos y producción se mantienen constantes, lo cual puede que ocurra en el corto plazo pero no así en periodos más largos.

Dicha relación puede variar en función de cambios en la productividad, choques externos, entre otros. Además, los modelos suponen que siempre existen recursos ociosos en la economía, con lo que cualquier incremento productivo es factible y constante, lo cual no siempre es cierto. No obstante, los resultados y pronósticos elaborados no dejan de ser útiles en la medida en que

Greivin Hernández González

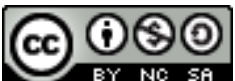

Revista Economía y Sociedad by Universidad Nacional is licensed under a CreativeCommons Reconocimiento-NoComercial- 
señalan de manera certera los sectores más importantes a considerar para la generación de empleo y la relación entre las variables de interés con alto grado de precisión.

Finalmente, cabe agregar que los resultados del estudio son en gran medida coincidentes con los de Sánchez (2014), en cuanto a los sectores con mayor potencial para la generación de empleo en términos de volumen, empleos indirectos y elasticidad. Las diferencias se pueden explicar por el grado de desagregación de los estudios, así como en el año base utilizado.

\section{Referencias}

Banco Central de Costa Rica (2016). Matriz simétrica de insumo-producto de la economía total por producto. Recuperado de http://www.bccr.fi.cr/estadisticas macro 2012/index.html

Crespi, G., Fernández-Arias, E., \& Stein, E. (Eds.). (2014). ¿Cómo repensar el desarrollo productivo? Políticas e instituciones sólidas para la transformación económica. Recuperado de https://publications.iadb.org/bitstream/handle/11319/6634/\%C2\%BFC\%C3\%B3mo\%20r epensar\%20el\%20desarrollo\%20productivo\%3F\%20Pol\%C3\%ADticas\%20e\%20institucion es\%20s\%C3\%B3lidas\%20para\%20la\%20transformaci\%C3\%B3n\%20econ\%C3\%B3mica.pdf ?sequence=1

Hernández, G., y Villalobos, C. (2016). Diversificación de la Matriz Productiva de Costa Rica ¿Alternativa para reducir dependencia, pobreza y desigualdad? Recuperado de http://library.fes.de/pdf-files/bueros/fesamcentral/13181.pdf

Instituto Nacional de Estadística y Censos. (2009). Encuesta de Hogares de Propósitos Múltiples. Recuperado de http://www.inec.go.cr/empleo

Instituto Nacional de Estadística y Censos. (2016). Encuesta Continua de Empleo (ECE). Recuperado de: http://www.inec.go.cr/empleo

Morales, R., Hernández, G., Salazar, G., \& Zárate, D. (2017). Situación del mercado laboral costarricense al tercer trimestre de 2016. Heredia: ESEUNA. Recuperado de https://drive.google.com/file/d/OB5ZkDQoWcO25Tk9RTFNZcjVseUU/view

REPRETEL. (Viernes 5 de febrero del 2016). Faltan 8 mil informáticos en el país. Recuperado de http://www.repretel.com/actualidad/faltan-8-mil-informaticos-en-el-pais-18275

40

Greivin Hernández González

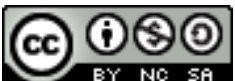

Revista Economía y Sociedad by Universidad Nacional is licensed under a CreativeCommons Reconocimiento-NoComercial- 
Salazar-Xirinachs, J., Nubler, I., y Kozul-Wrigth, R. (2014). Transforming Economies: making industrial policy work for growth, jobs and development. Recuperado de http://www.ilo.org/wcmsp5/groups/public/---dgreports/---dcomm/--publ/documents/publication/wcms_242878.pdf

Sánchez, M. (2014). Identificación de sectores económicos con alto potencial en la generación de empleo a partir de la matriz de insumo producto para Costa Rica 2011. Recuperado de http://studylib.es/doc/8281808/identificaci\%C3\%B3n-de-sectores-econ\%C3\%B3micoscon-alto-potencial- 


\section{ANEXO}

Tabla 1.

Costa Rica. Empleo directo e indirecto (número de ocupados) y elasticidad empleo-producto, según actividad económica, 2012.

\begin{tabular}{|c|c|c|c|c|}
\hline Actividad económica & $\begin{array}{l}\text { Empleo } \\
\text { directo }\end{array}$ & $\begin{array}{l}\text { Empleo } \\
\text { indirecto }\end{array}$ & $\begin{array}{c}\text { Empleo } \\
\text { total }\end{array}$ & Elasticidad \\
\hline Comercio & 385516 & 373962 & 759478 & 0,365 \\
\hline Construcción de edificios & 96284 & 261844 & 358128 & 0,172 \\
\hline Actividades de servicio de comida y bebidas & 74308 & 184896 & 259205 & 0,125 \\
\hline Enseñanza & 148039 & 72509 & 220548 & 0,106 \\
\hline Actividades de atención de la salud humana y de asistencia social & 83729 & 70412 & 154141 & 0,074 \\
\hline Actividades especializadas de las construcción & 36085 & 112391 & 148476 & 0,071 \\
\hline $\begin{array}{l}\text { Actividades de los hogares en calidad de empleadores de } \\
\text { personal doméstico }\end{array}$ & 145128 & 0 & 145128 & 0,070 \\
\hline $\begin{array}{l}\text { Elaboración y conservación de carne y embutidos de aves, de } \\
\text { ganado vacuno y porcino y otros tipos de carne }\end{array}$ & 14275 & 125356 & 139631 & 0,067 \\
\hline $\begin{array}{l}\text { Construcción de obras de servicio público y de otras de ingeniería } \\
\text { civil }\end{array}$ & 14641 & 91252 & 105893 & 0,051 \\
\hline Actividades de alojamiento & 32182 & 71999 & 104181 & 0,050 \\
\hline Cría de ganado vacuno & 36781 & 60989 & 97770 & 0,047 \\
\hline Elaboración de café oro & 2146 & 90929 & 93075 & 0,045 \\
\hline Cultivo de banano & 45396 & 45017 & 90413 & 0,043 \\
\hline Transporte por vía marítima, aérea y de carga por carretera & 25716 & 57302 & 83018 & 0,040 \\
\hline Elaboración de productos lácteos & 12507 & 70386 & 82893 & 0,040 \\
\hline Cultivo de piña & 27103 & 53807 & 80910 & 0,039 \\
\hline Mantenimiento y reparación de vehículos automotores & 40599 & 38460 & 79059 & 0,038 \\
\hline Actividad de intermediación monetaria & 28075 & 49754 & 77829 & 0,037 \\
\hline $\begin{array}{l}\text { Actividades administrativas y de apoyo de oficina y otras } \\
\text { actividades de apoyo a las empresas }\end{array}$ & 45074 & 31831 & 76906 & 0,037 \\
\hline Actividades inmobiliarias & 15624 & 58499 & 74123 & 0,036 \\
\hline Cultivo de café & 36101 & 37598 & 73699 & 0,035 \\
\hline $\begin{array}{l}\text { Administración del estado y aplicación de la política económica y } \\
\text { social de la comunidad }\end{array}$ & 34833 & 37511 & 72344 & 0,035 \\
\hline Transporte de pasajeros por taxi & 27932 & 32915 & 60847 & 0,029 \\
\hline Elaboración de productos de panadería y tortillas & 13029 & 46431 & 59460 & 0,029 \\
\hline Procesamiento y conservación de frutas y vegetales & 9015 & 42903 & 51918 & 0,025 \\
\hline Actividades de telecomunicaciones & 11693 & 39539 & 51232 & 0,025 \\
\hline Fabricación de instrumentos y suministros médicos y dentales & 12323 & 37388 & 49711 & 0,024 \\
\hline Prestación de servicios a la comunidad en general & 34191 & 12639 & 46830 & 0,023 \\
\hline Cultivo de otras hortalizas, raíces o tubérculos n.c.p. & 21616 & 24685 & 46301 & 0,022 \\
\hline Actividades de peluquería y otros tratamientos de belleza & 20444 & 24935 & 45379 & 0,022 \\
\hline Transporte terrestre de pasajeros excepto taxis & 18489 & 25006 & 43495 & 0,021 \\
\hline Actividades artísticas, de entretenimiento y recreativas & 27514 & 14021 & 41536 & 0,020 \\
\hline Suministro de energía eléctrica, gas, vapor y aire acondicionado & 12708 & 26814 & 39523 & 0,019 \\
\hline Publicidad y estudios de mercado & 12287 & 26776 & 39063 & 0,019 \\
\hline
\end{tabular}

42

Revista Economía y Sociedad by Universidad Nacional is licensed under a CreativeCommons Reconocimiento-NoComercial- 


\begin{tabular}{|c|c|c|c|c|}
\hline Actividad económica & $\begin{array}{l}\text { Empleo } \\
\text { directo }\end{array}$ & $\begin{array}{l}\text { Empleo } \\
\text { indirecto }\end{array}$ & $\begin{array}{c}\text { Empleo } \\
\text { total }\end{array}$ & Elasticidad \\
\hline Elaboración de azúcar & 3773 & 35291 & 39063 & 0,019 \\
\hline Elaboración de aceites y grasas de origen vegetal y animal & 2414 & 36259 & 38673 & 0,019 \\
\hline Fabricación de prendas de vestir & 15762 & 20933 & 36695 & 0,018 \\
\hline Cría de pollos & 6538 & 29968 & 36506 & 0,018 \\
\hline Actividades de seguridad e investigación & 19415 & 15785 & 35200 & 0,017 \\
\hline Manipulación de carga y otras actividades de apoyo al transporte & 8727 & 25683 & 34410 & 0,017 \\
\hline Fabricación de papel y productos de papel & 6720 & 27358 & 34078 & 0,016 \\
\hline $\begin{array}{l}\text { Destilación, rectificación, mezcla de bebidas alcohólicas y vinos / } \\
\text { Elaboración de bebidas malteadas, de malta, bebidas no } \\
\text { alcohólicas, aguas minerales, y otras aguas embotelladas / } \\
\text { Elaboración de productos de tabaco }\end{array}$ & 5620 & 28131 & 33751 & 0,016 \\
\hline $\begin{array}{l}\text { Elaboración de comidas, platos preparados y otros productos } \\
\text { alimenticios }\end{array}$ & 5489 & 27654 & 33143 & 0,016 \\
\hline Fabricación de muebles & 12932 & 19387 & 32318 & 0,016 \\
\hline $\begin{array}{l}\text { Reparación de computadoras, efectos personales y enseres } \\
\text { domésticos }\end{array}$ & 16496 & 14820 & 31316 & 0,015 \\
\hline Actividades de empleo & 26453 & 3934 & 30387 & 0,015 \\
\hline $\begin{array}{l}\text { Actividades de sociedades de cartera, fondos y sociedades de } \\
\text { inversión y otras actividades de servicios financieros }\end{array}$ & 5372 & 24727 & 30099 & 0,014 \\
\hline $\begin{array}{l}\text { Actividades de apoyo a la agricultura, la ganadería y actividades } \\
\text { postcosecha }\end{array}$ & 10155 & 19908 & 30064 & 0,014 \\
\hline $\begin{array}{l}\text { Actividades de consultoría en gestión financiera, recursos } \\
\text { humanos, mercadeo, oficinas principales y afines }\end{array}$ & 11211 & 18139 & 29350 & 0,014 \\
\hline $\begin{array}{l}\text { Fabricación de plásticos y de caucho sintético en formas } \\
\text { primarias / Fabricación de productos de plástico }\end{array}$ & 7035 & 22140 & 29176 & 0,014 \\
\hline $\begin{array}{l}\text { Servicios de información, programación y consultoría } \\
\text { informática, edición de programas informáticos y afines }\end{array}$ & 13736 & 13736 & 27471 & 0,013 \\
\hline $\begin{array}{l}\text { Actividades de impresión, edición y reproducción de grabaciones } \\
\text { excepto de programas informáticos }\end{array}$ & 11412 & 15826 & 27238 & 0,013 \\
\hline Actividades jurídicas & 11119 & 14909 & 26027 & 0,013 \\
\hline Construcción de carreteras y vías férreas & 5288 & 20476 & 25764 & 0,012 \\
\hline $\begin{array}{l}\text { Fabricación de productos elaborados de metal, excepto } \\
\text { maquinaria y equipo }\end{array}$ & 9331 & 15254 & 24585 & 0,012 \\
\hline Fabricación de equipo eléctrico y de maquinaria n.c.p. & 6727 & 17629 & 24356 & 0,012 \\
\hline Otras actividades profesionales, científicas y técnicas & 13518 & 10259 & 23778 & 0,011 \\
\hline $\begin{array}{l}\text { Actividades de arquitectura e ingeniería; ensayos y análisis } \\
\text { técnicos }\end{array}$ & 8482 & 15255 & 23738 & 0,011 \\
\hline Actividades limpieza general de edificios y de paisajismo & 17579 & 5030 & 22609 & 0,011 \\
\hline $\begin{array}{l}\text { Fabricación de cemento, cal, yeso y artículos de hormigón, } \\
\text { cemento y yeso y otros minerales no metálicos, n.c.p. }\end{array}$ & 3773 & 18806 & 22579 & 0,011 \\
\hline Reparación e instalación de maquinaria y equipo & 6461 & 16071 & 22532 & 0,011 \\
\hline $\begin{array}{l}\text { Procesamiento y conservación de pescados, crustáceos y } \\
\text { moluscos }\end{array}$ & 2756 & 19571 & 22327 & 0,011 \\
\hline Cultivo de caña de azúcar & 4106 & 18009 & 22115 & 0,011 \\
\hline $\begin{array}{l}\text { Elaboración de productos de molinería, excepto arroz, y } \\
\text { almidones y productos elaborados del almidón / Elaboración de } \\
\text { macarrones, fideos y productos farináceos análogos }\end{array}$ & 3578 & 18418 & 21996 & 0,011 \\
\hline
\end{tabular}

Revista Economía y Sociedad by Universidad Nacional is licensed under a CreativeCommons Reconocimiento-NoComercial- 


\begin{tabular}{|c|c|c|c|c|}
\hline Actividad económica & $\begin{array}{l}\text { Empleo } \\
\text { directo }\end{array}$ & $\begin{array}{l}\text { Empleo } \\
\text { indirecto }\end{array}$ & $\begin{array}{c}\text { Empleo } \\
\text { total }\end{array}$ & Elasticidad \\
\hline $\begin{array}{l}\text { Actividades de agencias de viajes, operadores turísticos, servicios } \\
\text { de reservas y actividades conexas }\end{array}$ & 6451 & 15380 & 21831 & 0,010 \\
\hline Cultivo de otras frutas, nueces y otros frutos oleaginosas & 10242 & 10986 & 21228 & 0,010 \\
\hline $\begin{array}{l}\text { Fabricación de jabones y detergentes, preparados para limpiar y } \\
\text { pulir, perfumes y preparados de tocador }\end{array}$ & 4366 & 16233 & 20598 & 0,010 \\
\hline $\begin{array}{l}\text { Actividades de contabilidad, teneduría de libros, consultoría } \\
\text { fiscal y otras actividades contables }\end{array}$ & 9730 & 10087 & 19817 & 0,010 \\
\hline $\begin{array}{l}\text { Producción de madera y fabricación de productos de madera y } \\
\text { corcho, excepto muebles; fabricación de artículos de paja y de } \\
\text { materiales transables }\end{array}$ & 6310 & 13274 & 19584 & 0,009 \\
\hline Actividades postales y de mensajería & 4179 & 14783 & 18962 & 0,009 \\
\hline Beneficio de arroz & 2223 & 16415 & 18638 & 0,009 \\
\hline Actividades de asociaciones & 2817 & 15772 & 18589 & 0,009 \\
\hline Cultivo de palma africana (aceitera) & 12013 & 5603 & 17616 & 0,008 \\
\hline $\begin{array}{l}\text { Actividades de alquiler y arrendamiento de activos tangibles e } \\
\text { intangibles no financieros }\end{array}$ & 6785 & 10486 & 17272 & 0,008 \\
\hline Otras industrias manufactureras & 4707 & 11722 & 16429 & 0,008 \\
\hline Fabricación de productos de caucho & 3146 & 12671 & 15817 & 0,008 \\
\hline Gestión de desechos y de descontaminación & 5563 & 9715 & 15278 & 0,007 \\
\hline Fabricación de metales comunes & 2311 & 12280 & 14591 & 0,007 \\
\hline Actividades de servicios vinculados al transporte & 4449 & 10137 & 14586 & 0,007 \\
\hline $\begin{array}{l}\text { Actividades auxiliares de servicios financieros, seguros y fondos } \\
\text { de pensiones }\end{array}$ & 4706 & 9383 & 14089 & 0,007 \\
\hline Fabricación de productos textiles & 4300 & 9530 & 13830 & 0,007 \\
\hline Suministro de agua potable y evacuación de aguas residuales & 6089 & 7182 & 13271 & 0,006 \\
\hline $\begin{array}{l}\text { Actividades de producción películas, videos y programas de } \\
\text { televisión, grabación de sonido, edición de música, programación } \\
\text { y transmisión }\end{array}$ & 3754 & 9100 & 12854 & 0,006 \\
\hline Producción de productos de café & 1110 & 11642 & 12752 & 0,006 \\
\hline $\begin{array}{l}\text { Fabricación de productos farmacéuticos, sustancias químicas } \\
\text { medicinales y de productos botánicos }\end{array}$ & 3409 & 9262 & 12671 & 0,006 \\
\hline Cultivo de frijol & 5306 & 7331 & 12637 & 0,006 \\
\hline $\begin{array}{l}\text { Actividad de seguros, reaseguros y fondos de pensiones, excepto } \\
\text { los planes de seguridad social de afiliación obligatoria }\end{array}$ & 2057 & 10580 & 12637 & 0,006 \\
\hline Elaboración de alimentos preparados para animales & 1552 & 10962 & 12514 & 0,006 \\
\hline Propagación de plantas & 5238 & 6373 & 11611 & 0,006 \\
\hline $\begin{array}{l}\text { Fabricación de componentes y tableros electrónicos, } \\
\text { computadoras y equipo periférico }\end{array}$ & 3480 & 7746 & 11226 & 0,005 \\
\hline Cultivo de arroz & 3691 & 7428 & 11119 & 0,005 \\
\hline Cría de cerdos & 1850 & 8816 & 10665 & 0,005 \\
\hline Extracción de piedra, arena y arcilla & 3540 & 7105 & 10645 & 0,005 \\
\hline Otras actividades de servicios n.c.p. & 5805 & 4552 & 10357 & 0,005 \\
\hline Cultivo de flores & 3487 & 5671 & 9158 & 0,004 \\
\hline Actividades de investigación científica y desarrollo & 3640 & 5270 & 8910 & 0,004 \\
\hline $\begin{array}{l}\text { Fabricación de los productos de la refinación del petróleo y de } \\
\text { coque / Fabricación de sustancias químicas básicas, abonos y }\end{array}$ & 1136 & 7176 & 8311 & 0,004 \\
\hline
\end{tabular}

44

Greivin Hernández González

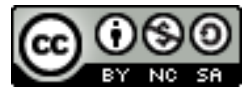

Revista Economía y Sociedad by Universidad Nacional is licensed under a CreativeCommons Reconocimiento-NoComercial- 
Economía y Sociedad, Vol. 22, № 52, julio-diciembre 2017, pp. 25-47

EISSN: 2215-3403 URL http://www.revistas.una.ac.cr/economia

Economía USOCIEDAD

\begin{tabular}{|c|c|c|c|c|}
\hline Actividad económica & $\begin{array}{l}\text { Empleo } \\
\text { directo }\end{array}$ & $\begin{array}{c}\text { Empleo } \\
\text { indirecto }\end{array}$ & $\begin{array}{c}\text { Empleo } \\
\text { total }\end{array}$ & Elasticidad \\
\hline \multicolumn{5}{|l|}{$\begin{array}{l}\text { compuestos de nitrógeno / Fabricación de otros productos } \\
\text { químicos n.c.p. y de fibras manufacturadas }\end{array}$} \\
\hline Cultivo de follajes & 2521 & 5206 & 7727 & 0,004 \\
\hline $\begin{array}{l}\text { Fabricación de pesticidas y de otros productos químicos de uso } \\
\text { agropecuario }\end{array}$ & 1906 & 5674 & 7580 & 0,004 \\
\hline $\begin{array}{l}\text { Fabricación de pinturas, barnices y productos de revestimiento } \\
\text { similares, tintas de imprenta y masillas }\end{array}$ & 1191 & 5948 & 7140 & 0,003 \\
\hline Acuicultura marítima y de agua dulce & 1485 & 5515 & 7000 & 0,003 \\
\hline Cría de otros animales & 3506 & 3490 & 6996 & 0,003 \\
\hline Cultivo de papa & 2318 & 4375 & 6693 & 0,003 \\
\hline Almacenamiento y depósito & 1715 & 4883 & 6598 & 0,003 \\
\hline Fabricación de vidrio y de productos de vidrio & 1056 & 5510 & 6566 & 0,003 \\
\hline Silvicultura y extracción de madera y caza & 4766 & 1452 & 6219 & 0,003 \\
\hline Fabricación de cuero y productos conexos excepto calzado & 1326 & 4888 & 6214 & 0,003 \\
\hline Cultivo de otras plantas no perennes y perennes & 2580 & 3566 & 6146 & 0,003 \\
\hline Pesca marítima y de agua dulce & 2332 & 3292 & 5624 & 0,003 \\
\hline Cultivo de maíz & 2017 & 3438 & 5456 & 0,003 \\
\hline Cultivo de plátano & 3002 & 2219 & 5221 & 0,003 \\
\hline Cultivo de melón & 1073 & 4138 & 5211 & 0,003 \\
\hline Elaboración de cacao, chocolate y productos de confitería & 1107 & 3847 & 4954 & 0,002 \\
\hline Cultivo de cebolla & 1890 & 2722 & 4612 & 0,002 \\
\hline $\begin{array}{l}\text { Fabricación de productos refractarios, materiales de } \\
\text { construcción de arcilla y de otros productos de porcelana y } \\
\text { cerámica }\end{array}$ & 1257 & 2913 & 4170 & 0,002 \\
\hline $\begin{array}{l}\text { Fabricación de vehículos automotores, remolques y } \\
\text { semirremolques / Fabricación de otros tipos de equipos de } \\
\text { transporte }\end{array}$ & 1362 & 2295 & 3657 & 0,002 \\
\hline Cultivo de sandía & 1054 & 2388 & 3441 & 0,002 \\
\hline Actividades veterinarias & 1273 & 2129 & 3402 & 0,002 \\
\hline Fabricación de productos de electrónica y de óptica & 994 & 2234 & 3229 & 0,002 \\
\hline Cultivo de chayote & 1746 & 1463 & 3210 & 0,002 \\
\hline Fabricación de calzado & 956 & 1827 & 2783 & 0,001 \\
\hline Actividades de planes de seguridad social de afiliación obligatoria & 563 & 2069 & 2632 & 0,001 \\
\hline Cultivo de otros cereales, legumbres y semillas oleaginosas n.c.p. & 1030 & 1293 & 2323 & 0,001 \\
\hline Actividades de funerales y actividades conexas & 506 & 889 & 1395 & 0,001 \\
\hline $\begin{array}{l}\text { Actividades de lavado y secado limpieza de prendas de tela y de } \\
\text { piel }\end{array}$ & 507 & 493 & 1000 & 0,000 \\
\hline Explotación de otras minas y canteras n.c.p. & 300 & 568 & 867 & 0,000 \\
\hline Transporte por ferrocarril & 16 & 222 & 238 & 0,000 \\
\hline Extracción de sal & 85 & 21 & 106 & 0,000 \\
\hline
\end{tabular}

Revista Economía y Sociedad by Universidad Nacional is licensed under a CreativeCommons Reconocimiento-NoComercial- 
Tabla 2.

Costa Rica: Generación de empleo indirecto según actividad económica, primer cuartil 2012. (Porcentaje del empleo indirecto dentro del empleo total)

\begin{tabular}{|c|c|}
\hline Actividad Económica & $\begin{array}{l}\text { \% empleo } \\
\text { indirecto }\end{array}$ \\
\hline Elaboración de café oro & $98 \%$ \\
\hline Elaboración de aceites y grasas de origen vegetal y animal & $94 \%$ \\
\hline Transporte por ferrocarril & $93 \%$ \\
\hline Producción de productos de café & $91 \%$ \\
\hline Elaboración de azúcar & $90 \%$ \\
\hline Elaboración y conservación de carne y embutidos de aves, de ganado vacuno y porcino y otros tipos de carne & $90 \%$ \\
\hline Beneficio de arroz & $88 \%$ \\
\hline Procesamiento y conservación de pescados, crustáceos y moluscos & $88 \%$ \\
\hline Elaboración de alimentos preparados para animales & $88 \%$ \\
\hline $\begin{array}{l}\text { Fabricación de los productos de la refinación del petróleo y de coque / Fabricación de sustancias químicas } \\
\text { básicas, abonos y compuestos de nitrógeno / Fabricación de otros productos químicos n.c.p. y de fibras } \\
\text { manufacturadas }\end{array}$ & $86 \%$ \\
\hline Construcción de obras de servicio público y de otras de ingeniería civil & $86 \%$ \\
\hline Elaboración de productos lácteos & $85 \%$ \\
\hline Actividades de asociaciones & $85 \%$ \\
\hline Fabricación de metales comunes & $84 \%$ \\
\hline Fabricación de vidrio y de productos de vidrio & $84 \%$ \\
\hline $\begin{array}{l}\text { Elaboración de productos de molinería, excepto arroz, y almidones y productos elaborados del almidón / } \\
\text { Elaboración de macarrones, fideos y productos farináceos análogos }\end{array}$ & $84 \%$ \\
\hline $\begin{array}{l}\text { Actividad de seguros, reaseguros y fondos de pensiones, excepto los planes de seguridad social de afiliación } \\
\text { obligatoria }\end{array}$ & $84 \%$ \\
\hline Elaboración de comidas, platos preparados y otros productos alimenticios & $83 \%$ \\
\hline $\begin{array}{l}\text { Destilación, rectificación, mezcla de bebidas alcohólicas y vinos / Elaboración de bebidas malteadas, de } \\
\text { malta, bebidas no alcohólicas, aguas minerales, y otras aguas embotelladas / Elaboración de productos de } \\
\text { tabaco }\end{array}$ & $83 \%$ \\
\hline Fabricación de pinturas, barnices y productos de revestimiento similares, tintas de imprenta y masillas & $83 \%$ \\
\hline $\begin{array}{l}\text { Fabricación de cemento, cal, yeso y artículos de hormigón, cemento y yeso y otros minerales no metálicos, } \\
\text { n.c.p. }\end{array}$ & $83 \%$ \\
\hline Cría de cerdos & $83 \%$ \\
\hline Procesamiento y conservación de frutas y vegetales & $83 \%$ \\
\hline $\begin{array}{l}\text { Actividades de sociedades de cartera, fondos y sociedades de inversión y otras actividades de servicios } \\
\text { financieros }\end{array}$ & $82 \%$ \\
\hline Cría de pollos & $82 \%$ \\
\hline Cultivo de caña de azúcar & $81 \%$ \\
\hline
\end{tabular}

46 


\section{Tabla 3.}

Costa Rica: Costo por empleo generado según actividad, primer cuartil, 2012 (millones de colones)

\begin{tabular}{|c|c|}
\hline Actividad económica & $\begin{array}{c}\text { Costo por empleo } \\
\text { generado }\end{array}$ \\
\hline Cultivo de frijol & 1,2 \\
\hline Cultivo de maíz & 1,3 \\
\hline Actividades de los hogares en calidad de empleadores de personal doméstico & 1,9 \\
\hline Extracción de sal & 2,1 \\
\hline Cultivo de otras hortalizas, raíces o tubérculos n.c.p. & 2,2 \\
\hline Cultivo de café & 2,5 \\
\hline Cultivo de papa & 2,8 \\
\hline Cría de otros animales & 2,8 \\
\hline Reparación de computadoras, efectos personales y enseres domésticos & 3,0 \\
\hline Elaboración de café oro & 3,1 \\
\hline Cultivo de otras frutas, nueces y otros frutos oleaginosas & 3,1 \\
\hline Cultivo de plátano & 3,2 \\
\hline Cultivo de follajes & 3,3 \\
\hline Fabricación de cuero y productos conexos excepto calzado & 3,3 \\
\hline Cultivo de flores & 3,4 \\
\hline Cultivo de otras plantas no perennes y perennes & 3,4 \\
\hline Cultivo de cebolla & 3,5 \\
\hline Explotación de otras minas y canteras n.c.p. & 3,5 \\
\hline Actividades de empleo & 3,6 \\
\hline Cría de ganado vacuno & 3,7 \\
\hline Actividades de peluquería y otros tratamientos de belleza & 3,9 \\
\hline Fabricación de calzado & 3,9 \\
\hline Cultivo de sandía & 4,0 \\
\hline Actividades de servicio de comida y bebidas & 4,0 \\
\hline Pesca marítima y de agua dulce & 4,1 \\
\hline Cultivo de caña de azúcar & 4,1 \\
\hline Cría de pollos & 4,2 \\
\hline
\end{tabular}

